\title{
NEW CFOA-BASED GROUNDED-CAPACITOR SINGLE-ELEMENT-CONTROLLED SINUSOIDAL OSCILLATOR
}

\author{
MUHAMMAD TAHER ABUELMA'ATTI* and \\ SA'AD MUHAMMAD AL-SHAHRANI
}

King Fahd University of Petroleum and Minerals, Box 203 Dhahran 31261 Saudi Arabia

(Received 24 June 1996; In final form 30 August 1996)

\begin{abstract}
A new grounded-capacitor single-element-controlled CFOA-based sinusoidal oscillator circuit is presented. The circuit exploits to advantage the internal pole of a general CFOA and uses externally connected three resistors and two grounded capacitors. The frequency of oscillation and the condition of oscillation of the proposed circuit can be independently controlled. Experimental results are included.
\end{abstract}

\section{INTRODUCTION}

At present there is a growing interest in using the current-feedback operational amplifier (CFOA) in various analog signal processing applications [1-7]. This is attributed to its larger bandwidth and higher slew rate compared to the conventional voltage feedback operational amplifier (VFOA). Thus, analog signal processing circuits built around the CFOA are expected to operate at higher frequencies than the VFOA-based circuits.

Recently, a number of CFOA-based sinusoidal oscillators have been proposed [8-12]. In some of these circuits, the CFOA pole is used to advantage. The CFOA-based RC-oscillators of References [8,9] exhibit

\footnotetext{
*Corresponding author.
} 
good performance but require equal-valued resistors and capacitors. In the active- $R$ multiphase sinusoidal oscillator of Reference [10], the frequency of oscillation and the condition of oscillation are coupled, thus a change in the frequency of oscillation cannot be achieved without disturbing the condition of oscillation. In the active- $R$ realizations of Reference [11], while the frequency of oscillation can be controlled without disturbing the condition of oscillation, by using a grounded (or a floating) resistor, the condition of oscillation cannot be adjusted without disturbing the frequency of oscillation. The partially active-R oscillator realization of Reference [11] uses only four passive elements. However, its frequency of oscillation cannot be controlled without disturbing the condition of oscillation. In Reference [12], a number of RC-oscillators using ideal CFOA and enjoying uncoupled frequency and condition of oscillation are proposed. However, in some circuits the control of the frequency or the condition of oscillation requires adjusting a floating resistor or a floating capacitor. Moreover, all the realizations [10-12] assume that terminal $\mathrm{Z}$ of the CFOA is accessible. This is not the case for all CFOAs. And thus, the proposed circuits can be realized using only the AD844 CFOA; other general CFOAs with no access to point $\mathrm{Z}$ cannot be used.

In this paper, a new grounded-capacitor CFOA-based sinusoidal oscillator circuit is presented. The proposed circuit utilizes to advantage the internal pole of a general CFOA and its frequency and condition of oscillation can be independently controlled. The proposed circuit can use any CFOA with, or without, access to point $\mathrm{Z}$.

\section{PROPOSED CIRCUIT}

Consider the oscillator structure shown in Fig. 1. A simplified equivalent circuit for the CFOA is shown in Fig. 2 [13]. In this equivalent circuit, $r_{i}$ represents the output resistance of the unity-gain buffers $A_{1}, C_{T}$ is the internally-connected compensation capacitor [14], and $R_{T}$ is the internal resistance of the gain node [15]. Assuming $v_{y}^{\prime}=\alpha v_{y}, i_{i n v}^{\prime}=\beta i_{i n v}$, $v_{z}^{\prime}=\gamma v_{z}$ where $\alpha=1-\epsilon_{1},\left|\epsilon_{1}\right| \ll 1$ represents the input voltage tracking error, $\beta=1-\epsilon_{2},\left|\epsilon_{2}\right| \ll 1$ represents the current tracking error, $\gamma=1-$ $\epsilon_{3},\left|\epsilon_{3}\right| \ll 1$ represents the output-voltage tracking error, routine analysis 


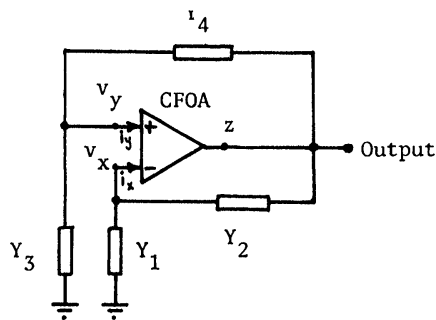

FIGURE 1 Proposed oscillator structure.

yields the characteristic equation of the circuit of Fig. 1, which can be expressed as

$$
\alpha \beta \gamma\left(Y_{1}+Y_{2}\right) Y_{4}=\left(Y_{3}+Y_{4}\right)\left(Y_{T}\left(1+r_{i}\left(Y_{1}+Y_{2}\right)\right)+\beta \gamma Y_{2}\right)
$$

where $Y_{T}=G_{T}+s C_{T}$ and $G_{T}=1 / R_{T}$.

Using (1), several oscillator circuits can be derived. In deriving these oscillator circuits, it must be noted that pure capacitive feedback between the output and the inverting input of the CFOA is not allowed [16]. Thus, a resistive feedback path must exist in order to ensure predictable operation of the oscillator circuit. If the admittances $Y_{1}, Y_{2}, Y_{3}$, and $Y_{4}$ are chosen such that $Y_{1}=G_{1}+s C_{1}, Y_{2}=G_{2}, Y_{3}=G_{3}+s C_{3}, Y_{4}=G_{4}$, a sinusoidal oscillator, shown in Fig. 3, can be obtained. Substituting these values of $Y_{1}-Y_{4}$ into (1), equating the real and imaginary parts of the resulting equation to zero, complex expressions for the frequency and the

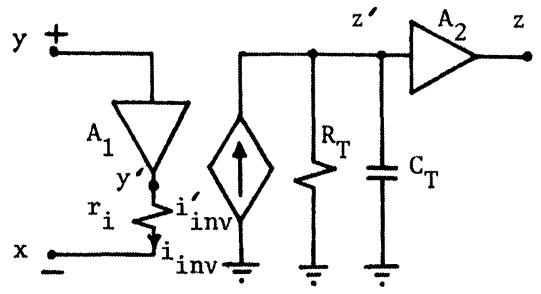

FIGURE 2 Simplified equivalent circuit for the CFOA. 


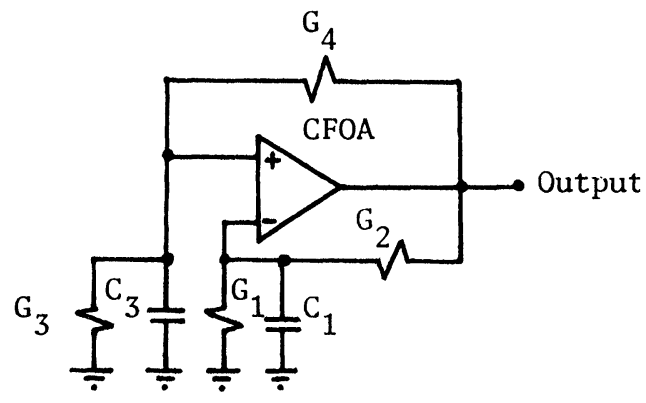

FIGURE 3 Proposed oscillator circuit obtained from Fig. 1.

condition of oscillation of the oscillator circuit of Fig. 3 can be obtained. However, simplified expressions for the frequency and the condition of oscillation can be obtained if the admittances $Y_{1}$ and $Y_{2}$ are chosen such that $r_{i}\left(Y_{1}+Y_{2}\right) \ll 1$. Thus, the frequency and the condition of oscillation of this oscillator circuit can be expressed by

$$
\omega_{0}^{2}=\frac{\left(G_{3}+G_{4}\right)\left(G_{T}+\beta \gamma G_{2}\right)-\alpha \beta \gamma G_{4}\left(G_{1}+G_{2}\right)}{C_{T} C_{3}}
$$

and

$$
\alpha \beta \gamma G_{4} C_{1}=\left(G_{3}+G_{4}\right) C_{T}+\left(G_{T}+\beta \gamma G_{2}\right) C_{3}
$$

From (2) and (3), one can see that the frequency of oscillation can be adjusted by tuning the grounded resistor $R_{1}=1 / G_{1}$ without disturbing the condition of oscillation. The condition of oscillation can be adjusted by tuning the grounded capacitor $C_{1}$ without disturbing the frequency of oscillation. Thus, the circuit enjoys independent control of the frequency and the condition of oscillation.

\section{EXPERIMENTAL RESULTS}

The proposed sinusoidal oscillator circuit was experimentally tested using the AD844 CFOA. Fig. 4 shows the results obtained with $R_{2}=738 \Omega, R_{3}$ $=197 \Omega, R_{4}=1.054 \mathrm{k} \Omega, C_{1}=200 \mathrm{pF}$, and $C_{3}=100 \mathrm{pF}$. For the AD844, 


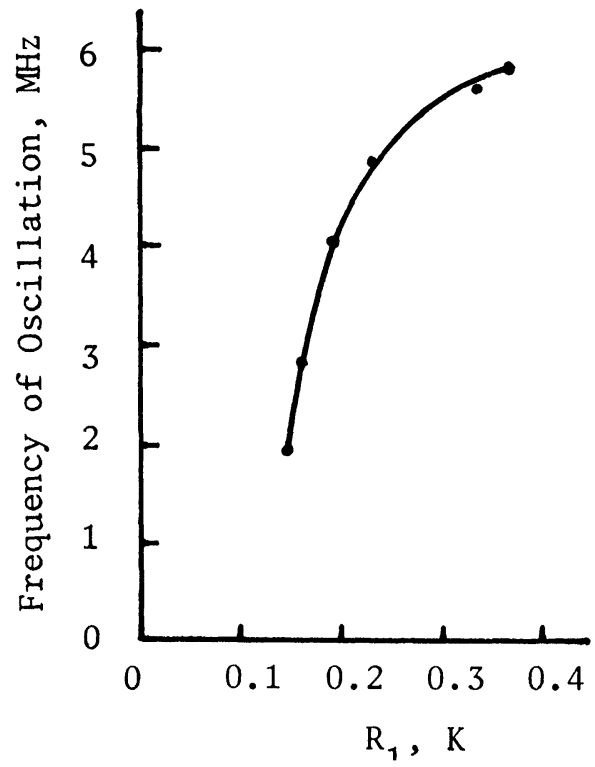

FIGURE 4 Measured frequency of oscillation with $R_{2}=738 \Omega, R_{3}=197 \Omega, R_{4}=1054$ $\Omega, C_{1}=200 P F, C_{3}=100 P F$.

the capacitance $C_{T} \cong 4.5 p F$ and the resistance $R_{T}=3 M \Omega$. However, the total parasitic capacitance between terminal $\mathrm{Z}$ of the AD844 and the breadboard was measured to be about $30 \mathrm{pF}$. Thus, $C_{T}$ was taken as $30 \mathrm{pF}$.

\section{CONCLUSION}

A new grounded-capacitor CFOA-based sinusoidal oscillator circuit has been presented. While the frequency of oscillation of the circuit can be changed by tuning a grounded resistor without disturbing the condition of oscillation, the condition of oscillation can be changed by tuning a grounded capacitor without disturbing the frequency of oscillation. This paves the way to electronic tuning of the frequency of oscillation as well as to automatic amplitude control. The use of grounded capacitors is another attractive feature for integration. Finally, it is worth mentioning that, in contrast with available CFOA-based oscillators, the implementation of 
the proposed circuit does not require access to point $\mathrm{Z}$ of the $\mathrm{CFOA}$. Thus, the AD844 as well as any general CFOA with no access to point $\mathrm{Z}$, can be used for implementing the proposed oscillator circuit.

\section{References}

[1] Analog Devices, Linear Products Data Book, Norwood, Massachusetts: Analog Devices, (1990)

[2] Comlinear Corp., Application Note 200-1. Designer's Guide for 200 seies op-amp, Ft. Colls, Colorado: Comlinear Corp., (1984).

[3] Fabre, A. (1992). Gyrator implementation from commercially available transimpedance amplifiers, Electronics Letters, Vol. 28, pp. 263-264.

[4] Fabre, A. (1993). Insensitive voltage-mode and current-mode filters from commercially available transimpedance opamps, IEE Proceedings, Part G, Vol. 140, pp. 319321.

[5] Chang, C.-M., Hwang, C.-S. and Tu, S.-H. (1994). Voltage-mode notch, lowpass and bandpass filter using current-feedback amplifiers, Electronics Letters, Vol. 30, pp. 2022-2023.

[6] Chang, C.-M. and Hwang, C.-S. (1995). Comment on "Voltage-mode notch, lowpass and bandpass filter using current-feedback amplifiers," Electronics Letters, Vol. 31, p. 246.

[7] Liu, S.-I. and Chen, J.-J. (1995). Realisation of analogue divider using current feedback amplifiers, IEE Proceedings-Circuits Devices Systems, Vol. 142, pp. 45-48.

[8] Celma, S., Martinez, P. A. and Carlosena, A. (1994). Current feedback amplifiers based sinusoidal oscillators, IEEE Transactions Circuits and Systems-I: Fundamental Theories and Applications, Vol. 41, pp. 906-908.

[9] Celma, S., Carlosena, A. and Martinez, P. A. (1994). Current feedback amplifiers based sinusoidal oscillators, International Symposium on Circuits and Systems, Vol. 5, pp. 101-104.

[10] Wu, D.-S., Liu, S.-I., Hwang, Y.-S. and Wu, Y.-P. (1995). Multiphase sinusoidal oscillator using the CFOA pole, IEE Proceedings-Circuits Devices Systems, Vol. 142, pp. $3.7-40$.

[11] Liu, S.-I., Chang, C.-C. and Wu, D.-S. (1994). Active-R sinusoidal oscillators using the CFA pole, International Journal of Electronics, Vol. 77, pp. 1035-1042.

[12] Liu, S.-I., Shih, C.-S. and Wu, D.-S. (1994). Sinusoidal oscillators with single element control using a current-feedback amplifier, International Journal of Electronics, Vol. 77, pp. 1007-1013.

[13] Payne, A. and Toumazou, C. (1992). High frequency self-compensation of currentfeedback devices, International Symposium on Circuits and Systems, pp. 1376-1379.

[14] Bruun, E. (1994). A dual current feedback OpAmp in CMOS technology, Analog Integrated Circuits and Signal Processing, Vol. 5, pp. 213-217.

[15] Harvey, B. (1993). Current feedback opamp limitations: A state-of-the-art review, International Symposium on Circuits and Systems, pp. 1066-1069.

[16] Toumazou, C. and Lidgey, J. (1994). Current-feedback Op Amps: A blessing in disguise? IEEE Magazine of Circuits and Devices, Vol. 10, January, pp. 34-37. 

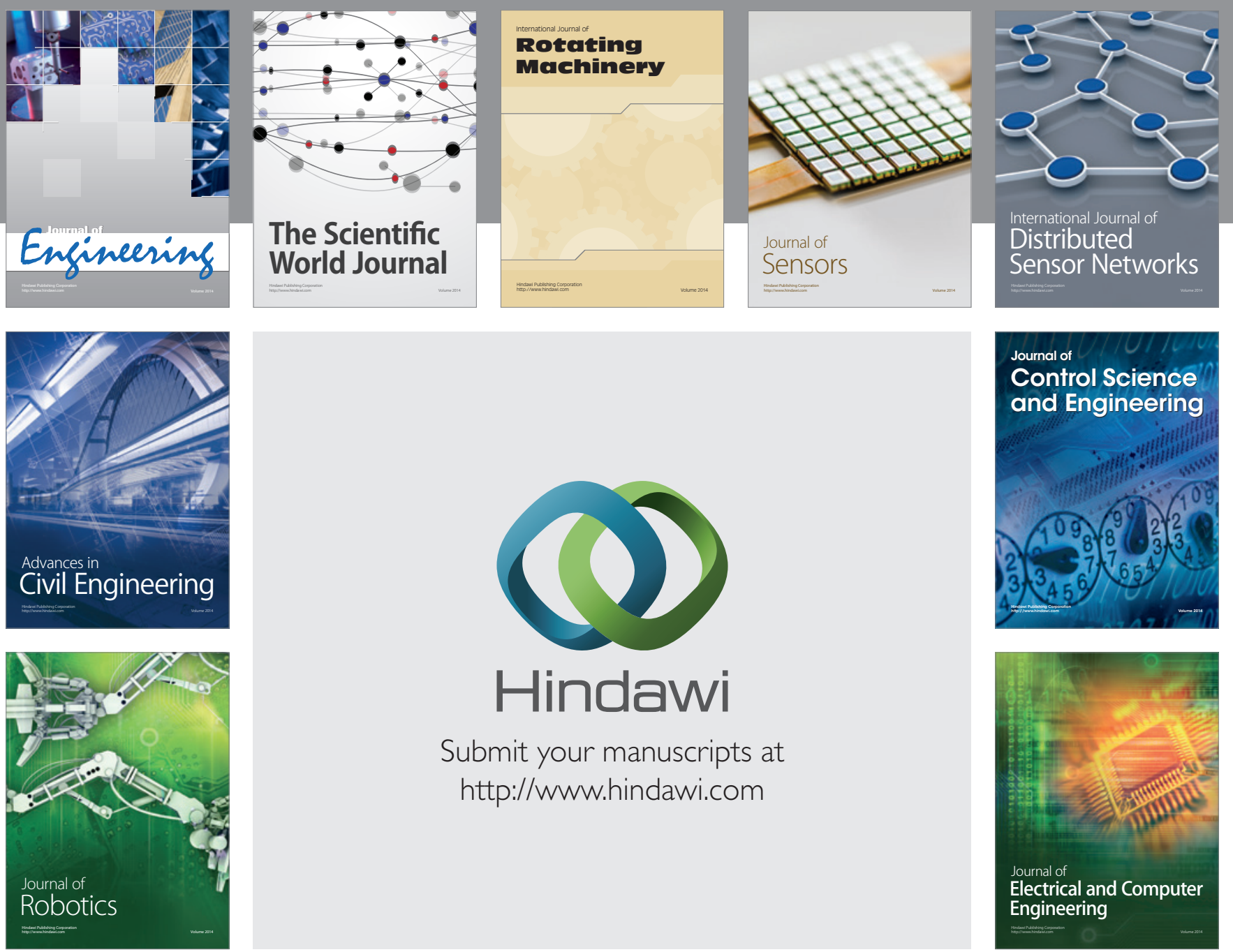

Submit your manuscripts at

http://www.hindawi.com
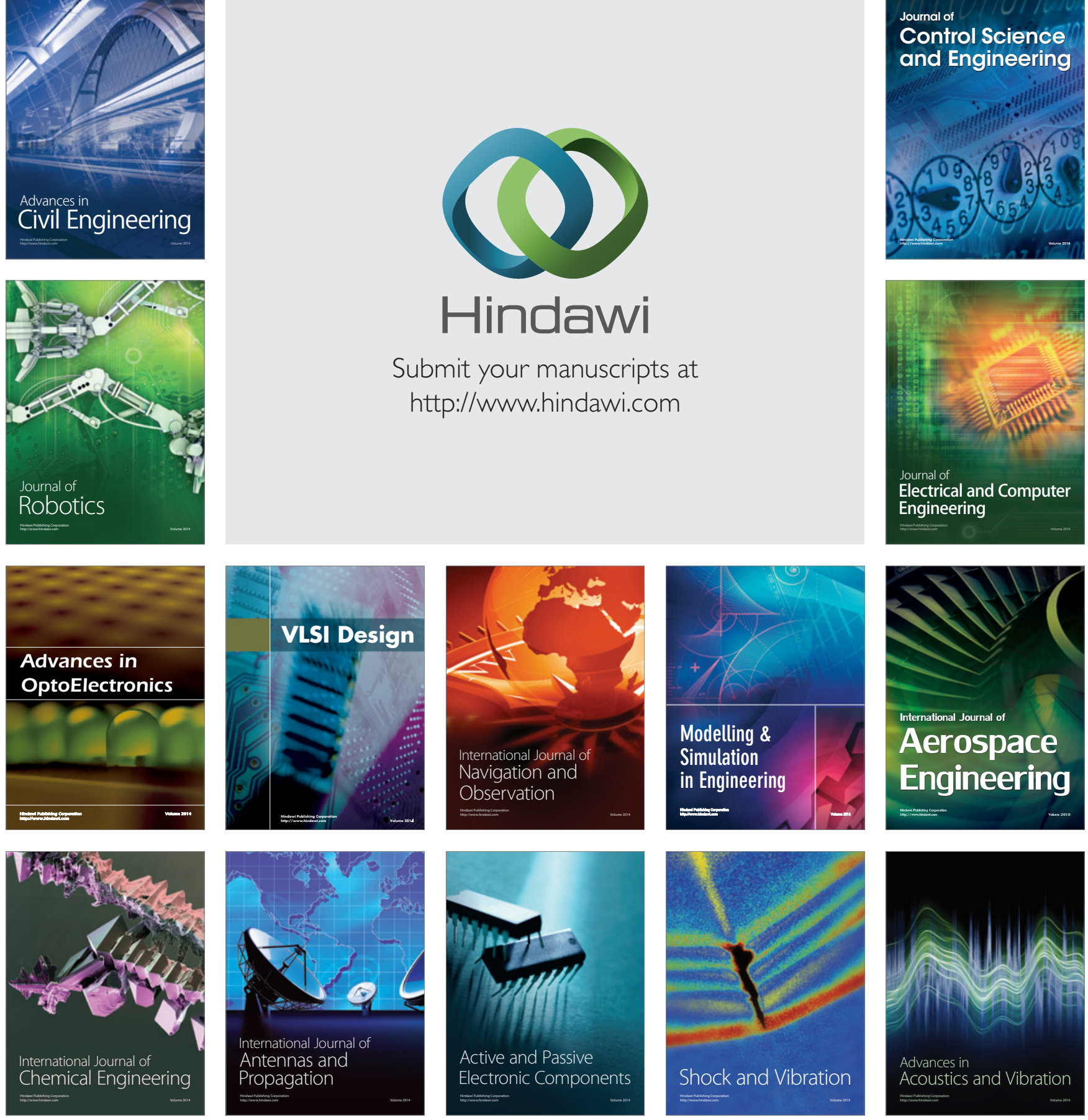